\title{
Bedside thromboelastography to rapidly assess the pharmacodynamic response of anticoagulants and aspirin in COVID-19: evidence of inadequate therapy in a predominantly minority population
}

\author{
Paul A. Gurbel ${ }^{1}$ D $\cdot$ Kevin P. Bliden ${ }^{1} \cdot$ Amit Rout $^{1} \cdot$ Nicole Rapista $^{1} \cdot$ Naval Walia $^{1} \cdot$ Rahul Chaudhary $^{2} \cdot$ Gordon Ens $^{3}$. \\ Malina Traianova ${ }^{4} \cdot$ Jaime S. Barnes ${ }^{5} \cdot$ Udaya S. Tantry $^{1}$
}

Accepted: 18 March 2021 / Published online: 7 April 2021

(c) The Author(s), under exclusive licence to Springer Science+Business Media, LLC, part of Springer Nature 2021

COVID-19 is a thromboinflammatory disease [1]. Anticoagulant prophylaxis is currently widely used to attenuate thrombotic complications, and aspirin has also been recently proposed as a potential therapeutic option [2, 3]. However, there is limited evidence of anticoagulants' pharmacodynamic efficacy, and no study of the pharmacodynamic efficacy of aspirin is available. No published experience with bedside assays to assess anticoagulant or antiplatelet response in COVID-19 is available.

Here, we report a sub-analysis of the evaluation of hemostasis in hospitalized COVID-19 patients (TARGETCOVID) study (URL: https://www.clinicaltrials.gov; Unique identifier: NCT04493307). The study was performed in accordance with standard ethical principles and approved by the local institutional review board. All patients provided written consent. We enrolled hospitalized patients diagnosed with COVID-19 by reverse transcription-polymerase chain reaction assay $(n=120)$ and patients without COVID-19 but with pneumonia and an elevated D-dimer $(n=13)$. Using a bedside thromboelastography assay (TEG-6s, Haemonetics Corporation, Braintree, MA, USA), we measured the reaction time (R) as an indicator of anticoagulant response and platelet function with the platelet mapping assay at the time

Paul A. Gurbel

pgurbel@lifebridgehealth.org

1 Sinai Center for Thrombosis Research and Drug Development, Sinai Hospital of Baltimore, Lifebridge Health, Baltimore, MD 21215, USA

2 Division of Hospital Internal Medicine, Mayo Clinic, Rochester, MN, USA

3 Inflammatory Markers Laboratory, Wichita, KS, USA

4 CEDEX Laboratory, Nashua, NH, USA

5 Critical Care Medicine, Sinai Hospital and Northwest Hospital, Lifebridge Health, Baltimore, MD, USA of hospital administration $[4,5]$. Aspirin effect was further determined by urinary 11-dehydro-thromboxane $\mathrm{B}_{2}$ (u11-dh $\mathrm{TxB}_{2}$ ) with enzyme-linked immune assay (Inflammatory Markers Laboratory, Wichita, KS, USA), total thrombus formation analysis system (T-TAS) with platelet chip (Fujimori Kogyo Co, Tokyo, Japan) and whole blood aggregometry (Chronolog Corporation, Havertown, PA, USA).

The majority of our COVID-19 positive patients were African Americans (67\%). The difference in $\mathrm{R}(\Delta \mathrm{R}) \geq 1 \mathrm{~min}$ between the kaolin and kaolin plus heparinase channels in the TEG6s indicated an anticoagulant effect [5]. Aspirin response was assessed in patients admitted on chronic aspirin therapy ( $\geq 14$ days) and anticoagulant therapy was assessed $\geq 24 \mathrm{~h}$ after administration.

Compared to patients on enoxaparin prophylaxis (subcutaneous enoxaparin $<80 \mathrm{mg}$ BID) $(\mathrm{n}=50)$, patients on heparin prophylaxis (subcutaneous unfractionated heparin $\leq 7500$ units TID) $(n=21)$ and therapeutic anticoagulation (intravenous unfractionated heparin or subcutaneous enoxaparin $\geq 80 \mathrm{mg}$ BID) $(\mathrm{n}=17)$ exhibited a significantly greater $\Delta \mathrm{R}(0.31 \pm 0.8$ versus $1.2 \pm 1.7$ and $1.5 \pm 1.4 \mathrm{~min}$, respectively, $\mathrm{p}<0.004$ for both comparisons). There was a higher incidence of poor anticoagulant response $(\Delta \mathrm{R}<1 \mathrm{~min})$ with enoxaparin prophylaxis compared to heparin prophylaxis and intravenous heparin (84\% versus $62 \%$ and $53 \%$, respectively, $\mathrm{p}<0.05$ for both comparisons) (Fig. 1a).

In total, $29 \%$ of patients were on aspirin therapy. With TEG-6s, 94\% of COVID-19 patients demonstrated $>50 \%$ platelet aggregation induced by $1 \mathrm{mmol} / \mathrm{L}$ arachidonic acid (AA) (aspirin nonresponsiveness) (data are not shown). In patients on aspirin, u11-dh $\mathrm{TxB}_{2}$ was similar in COVID19 positive and negative patients $(3760 \pm 2295$ versus $3051 \pm 1488 \mathrm{pg} / \mathrm{mg}$ creatinine). However, the number of non-COVID-19 patients were small to draw a definitive conclusion. In COVID-19 patients, u11-dh $\mathrm{TxB}_{2}$ was lower in aspirin-treated patients than patients not on aspirin 


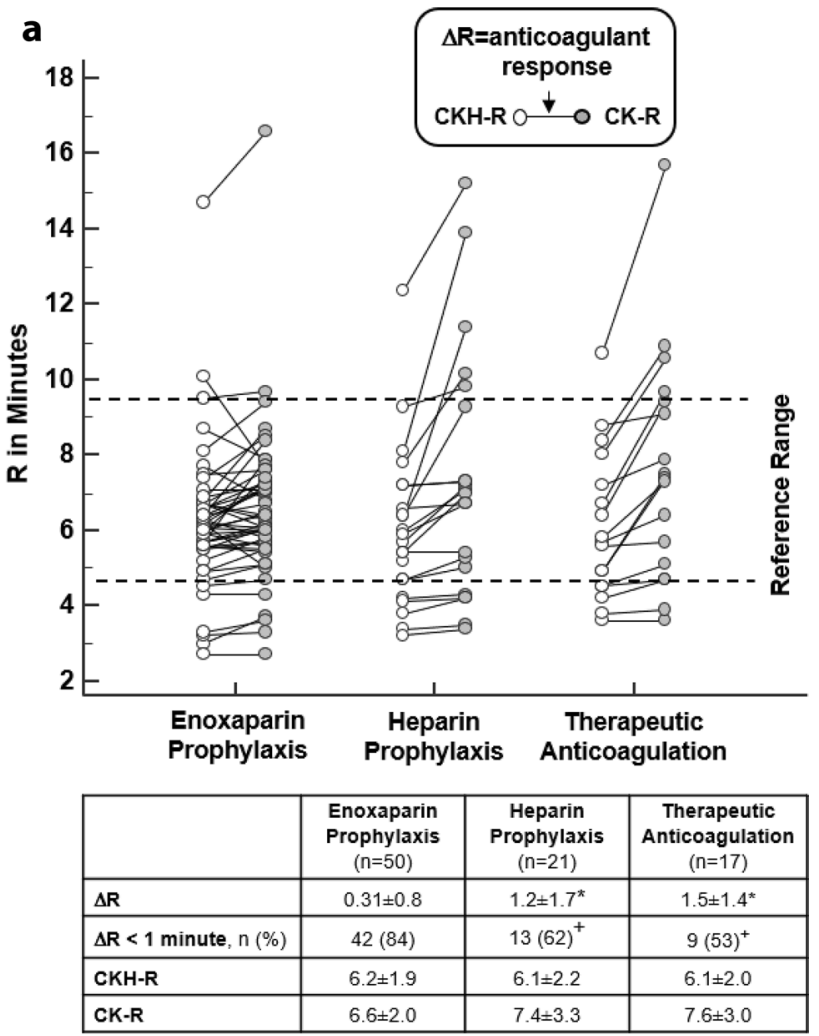

b

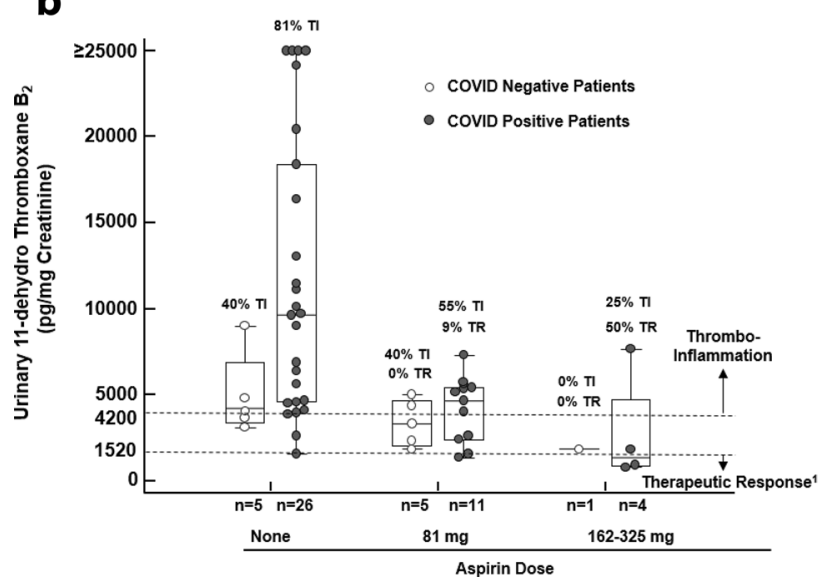

Fig. 1 a Anticoagulant response indicated by the change in reaction time by thromboelastography in patients with COVID-19. $R$ reaction time, $C K-R$ reaction time with kaolin channel, $C K H-R$ reaction time in kaolin plus heparinase channel. ${ }^{*} \mathrm{p}<0.004$ as compared to enoxaparin prophylaxis. $+\mathrm{p}<0.05$ as compared to enoxaparin prophylaxis. b Urinary 11-dehydro thromboxane $B_{2}$ in COVID-19 positive and negative patients with and without Aspirin. TI thromboinflammation, TR therapeutic response [6]

$(3760 \pm 2295$ versus $13,125 \pm 11,474 \mathrm{pg} / \mathrm{mg}$ creatinine, $\mathrm{p}=0.003)$. An inadequate therapeutic aspirin response was observed in $91 \%$ of COVID-19 patients on $81 \mathrm{mg}$ daily aspirin and $50 \%$ of patients on $\geq 162 \mathrm{mg}$ daily aspirin (Fig. 1b). The frequency of thromboinflammation was highest in COVID-19 patients not on aspirin (81\%) and lower on $81 \mathrm{mg}$ daily (55\%) and lowest on $\geq 162 \mathrm{mg}$ daily aspirin (25\%). Compared to COVID-19 patients not on aspirin, patients on $81 \mathrm{mg}$ daily aspirin exhibited a trend for lower whole blood aggregation in response to $5 \mu \mathrm{g} / \mathrm{mL}$ collagen $(7.6 \pm 4.1 \%$ versus $9.4 \pm 3.3 \%, \mathrm{p}=0.08)$ and $50 \mathrm{umol} / \mathrm{L}$ epinephrine $(5.0 \pm 4.3 \%$ versus $6.7 \pm 4.1 \%, p=0.1)$. With T-TAS platelet chip, COVID-19 patients on $81 \mathrm{mg}$ daily aspirin exhibited lower area under the curve compared to patients not on aspirin $(226 \pm 174$ versus $305 \pm 135, p=0.01)$. No significant correlation between arachidonic acid- or collagen-induced platelet aggregation and $\mathrm{u} 11-\mathrm{dh} \mathrm{TxB}_{2}$ was observed.

This is the first report of a point-of-care care assay demonstrating an inadequate pharmacodynamic response to anticoagulants and aspirin in a high percentage of COVID19 patients, most of whom were African Americans. An insufficient pharmacodynamic effect of $81 \mathrm{mg}$ daily aspirin indicated by high levels of $\mathrm{u} 11-\mathrm{dh} \mathrm{TxB}_{2}$ and area under the curve with platelet chip with T-TAS suggests that low dose aspirin is insufficient to provide a meaningful clinical effect in the presence of elevated systemic inflammation (cytokine storm) and hypercoagulability. Furthermore, most of the COVID-19 positive patients on anticoagulant therapy, particularly enoxaparin prophylaxis, may not achieve the desired pharmacodynamic effect attributed to high fibrinogen levels. Our results should be considered hypothesis-generating for personalizing antithrombotic therapy to improve outcomes in COVID-19. Extrapolation of our findings to other races and ethnicities will require further study. These findings are relevant to ongoing studies of antithrombotic therapy in COVID-19.

Acknowledgements Chronolog Corporation provided instrument and supplies for the whole blood aggregation, Hikari Dx provided supplies for the T-TAS assay, Haemonetics donated instrument and cartridges for thromboelastography and $\mathrm{u} 11-\mathrm{dh} \mathrm{TxB}_{2}$ samples were processed by Inflammatory Markers Laboratory.

Disclosures Dr. Gurbel reports grants and personal fees from Bayer HealthCare LLC, Otitopic Inc, Amgen, Janssen, and US WorldMeds LLC; grants from Instrumentation Laboratory, Haemonetics, Medicure Inc, Idorsia Pharmaceuticals, and Hikari Dx; personal fees from UpToDate; Dr Gurbel is a relator and expert witness in litigation involving clopidogrel; in addition, Dr. Gurbel has two patents, Detection of restenosis risk in patients issued and Assessment of cardiac health and thrombotic risk in a patient. Dr. Tantry reports receiving honoraria from UptoDate and Aggredyne. Other author reports no disclosures.

Funding Platelet and Thrombosis Research, LLC, Baltimore, MD, USA.

\section{References}

1. Chaudhary R, Kreutz RP, Bliden KP, Tantry US, Gurbel PA (2020) Personalizing antithrombotic therapy in COVID-19: role 
of thromboelastography and thromboelastometry. Thromb Haemost 120:1594-1596

2. Tang N, Bai H, Chen X, Gong J, Li D, Sun Z (2020) Anticoagulant treatment is associated with decreased mortality in severe coronavirus disease 2019 patients with coagulopathy. J Thromb Haemost 18:1094-1099

3. Gurbel PA, Bliden KP, Schrör K (2020) Can an old ally defeat a new enemy? Circulation 142:315-317

4. Gurbel PA, Bliden KP, Tantry US, Monroe AL, Muresan AA, Brunner NE, Lopez-Espina CG, Delmenico PR, Cohen E, Raviv G, Haugen DL, Ereth MH (2016) First report of the point-of-care TEG: a technical validation study of the TEG-6S system. Platelets 27:642-649

5. Van PY, Cho SD, Underwood SJ, Morris MS, Watters JM, Schreiber MA (2009) Thrombelastography versus AntiFactor Xa levels in the assessment of prophylactic-dose enoxaparin in critically ill patients. J Trauma 66:1509

6. Gurbel PA, Bliden KP, Tantry US (2021) Defining platelet response to acetylsalicylic acid: the relation between inhibition of serum thromboxane B2 and agonist-induced platelet aggregation. J Thromb Thrombolysis 51:260-264

Publisher's Note Springer Nature remains neutral with regard to jurisdictional claims in published maps and institutional affiliations. 